\title{
Tape casting of a YAG/Yb:YAG/YAG transparent ceramic for a broadband tunable laser
}

\author{
Chao Wang ${ }^{1}$, Wenxue $\mathrm{Li}^{1}$, Xianghui Yang ${ }^{1}$, Dongbi Bai ${ }^{1}$, Kangwen Yang ${ }^{1}$, Xuewei $\mathrm{Ba}^{2}, \mathrm{Jiang}^{\mathrm{Li}}{ }^{2}$, \\ Yubai Pan ${ }^{2}$, and Heping Zeng ${ }^{1}$ \\ ${ }^{1}$ State Key Laboratory of Precision Spectroscopy, East China Normal University, Shanghai 200062, China \\ ${ }^{2}$ Key Laboratory of Transparent Opt-functional Inorganic Materials, Shanghai Institute of Ceramics, Chinese Academy of Sciences, \\ Shanghai 200050, China \\ (Received 28 March 2014; revised 13 October 2014; accepted 22 October 2014)
}

\begin{abstract}
A composite transparent YAG/Yb:YAG/YAG ceramic was prepared by a non-aqueous tape-casting method. An optical transmittance of $82 \%$ was obtained at visible wavelength and around $1100 \mathrm{~nm}$. A low-threshold, broadband tunable continuous-wave $(\mathrm{CW})$ laser at $1031 \mathrm{~nm}$ was further demonstrated from the ceramic sample, which was pumped by a $974 \mathrm{~nm}$ fiber-pigtailed laser diode. The threshold pump power was $0.45 \mathrm{~W}$ and the maximum output power was $3.2 \mathrm{~W}$, corresponding to a slope efficiency of $20.4 \%$. By inserting an SF57 prism in the laser cavity, the output wavelength could be tuned continuously from 1021 to $1058 \mathrm{~nm}$.
\end{abstract}

Keywords: composite transparent ceramic; solid-state laser; broadband tunable wavelength

\section{Introduction}

Transparent ceramic materials have been widely used in building high-performance solid-state lasers due to their significant advantages over single-crystal materials such as large-size fabrication, reduced production cycle, high doping concentration and stable chemical properties ${ }^{[1-4]}$. Early research into potential substitutions of high-power laser gain media was focused on neodymium-doped YAG ceramics. In 1995, Ikesue demonstrated a 1.1 at.\% Nd:YAG ceramic laser for the first time and observed that the continuous-wave (CW) laser had a slope efficiency of $28 \%$ at $1060 \mathrm{~nm}^{[5]}$. In 2001, Lu demonstrated laser oscillation with 1.0 at.\% Nd:YAG ceramic and the output power reached $72 \mathrm{~W}^{[6]}$. One year later, a group led by Ueda increased the output power of a Nd:YAG ceramic laser to $1.46 \mathrm{~kW}$, corresponding to a slope efficiency of $42 \%{ }^{[7]}$. However, the gain bandwidth of the $\mathrm{Nd}^{3+}$ ion is merely $1 \mathrm{~nm}$, limiting the output pulse width to picosecond magnitude in mode-locked operation for the purpose of generating ultrafast lasers ${ }^{[8-10]}$.

Compared with $\mathrm{Nd}^{3+}, \mathrm{Yb}^{3+}$ has been recognized as a more attractive alternative for high-power or ultrashort-pulse laser operation due to its simple two-manifold energy levels

Correspondence to: W. Li, East China Normal University, State Key Laboratory of Precision Spectroscopy Room B113, Science Building, 3663 N. Zhongshan Road, Shanghai, CN 200062, China. Email: wxli@phy.ecnu.edu.cn and large emission cross-section, which is beneficial for the elimination of undesired effects such as up-conversion, cross relaxation and excited-state absorption ${ }^{[11-15]}$. In particular, the relatively broadband absorption cross-section of $\mathrm{Yb}$ ions enables high pump absorption efficiency from 941 to $970 \mathrm{~nm}$, which is within the emission wavelength range of high-power, low-cost InGaAs laser diodes, thus making diode-pumped solid-state $\mathrm{Yb}$-doped lasers attractive for practical high-power and high-energy applications ${ }^{[16-18]}$. Moreover, the large emission cross-section of $\mathrm{Yb}$ material enables high-efficiency and widely tunable laser operation $^{[19-22]}$.

However, with the substantial increase of output power in solid-state lasers, the heavy thermal load of the quasithree-level structure has become a severe problem that restricts the power scaling of $\mathrm{Yb}$-doped solid-state lasers. Therefore, the exploration of advanced materials with superior heat transfer performance has become a hot scientific focus. In 2006, Ikesue and Aung demonstrated the fabrication of a laser ceramic with a complicated structure without precise polishing and diffusion bonding; the advanced ceramic processing technique they presented paved the way to the design of laser gain media with flexibility and convenience ${ }^{[23]}$. Soon after, they carried out in-depth research into the advantages of composite-structure laser ceramic, which was reported in 2008. Through a comparison of the thermal distribution between a conventional ceramic 
with uniform doping concentration and a novel ceramic with smooth gradient doping concentration in an edge-pumped laser, the experimental results showed that thermal lensing effects were efficiently suppressed in the composite ceramic due to the excellent gradient distribution of doping ions ${ }^{[24]}$. Moreover, the composite ceramic structure also allows for effective beam mode and pattern control of Q-switched or mode-locked lasers, which will promote the integration and downsizing of solid-state laser oscillators ${ }^{[25-27]}$. Recently, a diode corner-pumped high-power slab laser with an output power of more than $520 \mathrm{~W}$ and a slope efficiency of $32 \%$ was introduced due to the easy synthesis of ceramic active media and a simple pumping system ${ }^{[28]}$.

In this paper, we report the fabrication of a multilayer composite YAG/5 at.\% Yb:YAG/YAG laser ceramic by a nonaqueous tape-casting and vacuum-sintering process. The transmittance characteristics and laser performance of this composite ceramic are demonstrated. The experimental results indicate that low-threshold and continually tunable properties are achieved under high-brightness diode laser pumping.

\section{Experimental setup and results}

The non-aqueous tape-casting technique was adopted in our study to fabricate multilayer ceramics. To begin with, we prepared two groups of high-purity commercial powders as starting materials. An $\mathrm{Al}_{2} \mathrm{O}_{3}$ and $\mathrm{Y}_{2} \mathrm{O}_{3}$ mixture was used to produce undoped $\mathrm{YAG}$ slurry, while an $\mathrm{Yb}_{2} \mathrm{O}_{3}, \mathrm{Al}_{2} \mathrm{O}_{3}$ and $\mathrm{Y}_{2} \mathrm{O}_{3}$ mixture was used to produce 5 at.\% Yb:YAG slurry. The weight of each powder was precisely controlled with a stoichiometric ratio of YAG and 5 at.\% Yb:YAG, respectively. We used polyvinyl butyral, PEG-400 and butyl benzyl phthalate as the binder and the plasticizers. The binder and the plasticizers were added into the powders and the mixture was then ball-milled for $10 \mathrm{~h}$. A degassing process was subsequently carried out in a vacuum device for $30 \mathrm{~min}$. After that, the tape-casting process was implemented with a height between the blade and the substrate of $350 \mu \mathrm{m}$ and a casting velocity of $100 \mathrm{~mm} \mathrm{~min}^{-1}$. The eight stacked tapes obtained had a thickness of approximately $1 \mathrm{~mm}$ and were clipped into wafers with a diameter of $20 \mathrm{~mm}$. We chose 15 undoped tapes as the bottom layer, stacked 10 doped tapes as the interlayer, and placed another 15 undoped tapes onto them as the top layer. These sandwich structure multi-tapes were laminated at $85^{\circ} \mathrm{C}$ for $30 \mathrm{~min}$ under a pressure of 40 MPa to obtain the green samples. The samples were sintered at $1760^{\circ} \mathrm{C}$ for $50 \mathrm{~h}$ under vacuum conditions at a pressure of less than $1 \times 10^{-3} \mathrm{~Pa}$ and annealed at $1450^{\circ} \mathrm{C}$ for $10 \mathrm{~h}$ in a stove to improve the optical quality of the ceramic samples.

Figure 1 shows photographs of YAG/Yb:YAG/YAG ceramic synthesized by the non-aqueous tape-casting method. The top image is the unannealed green body and the bottom image is the annealed laser ceramic. The color of the unannealed ceramic was green because of light absorption

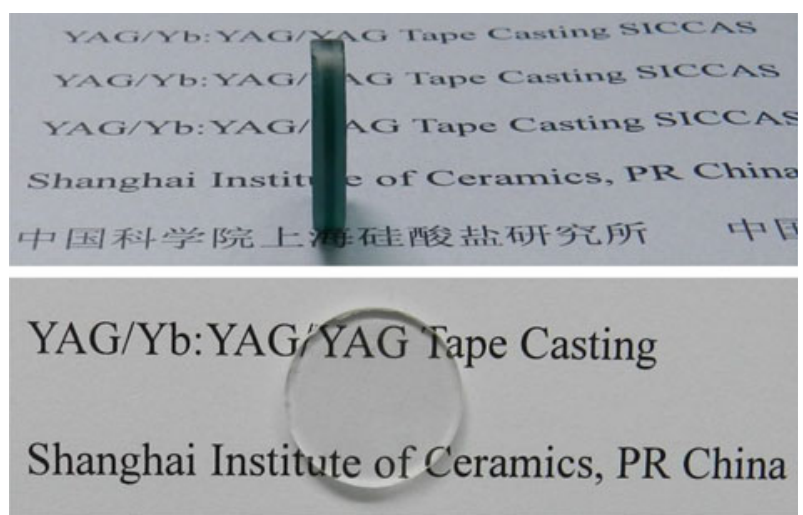

Figure 1. An image of the YAG/Yb:YAG/YAG (top: unannealed; bottom: annealed).

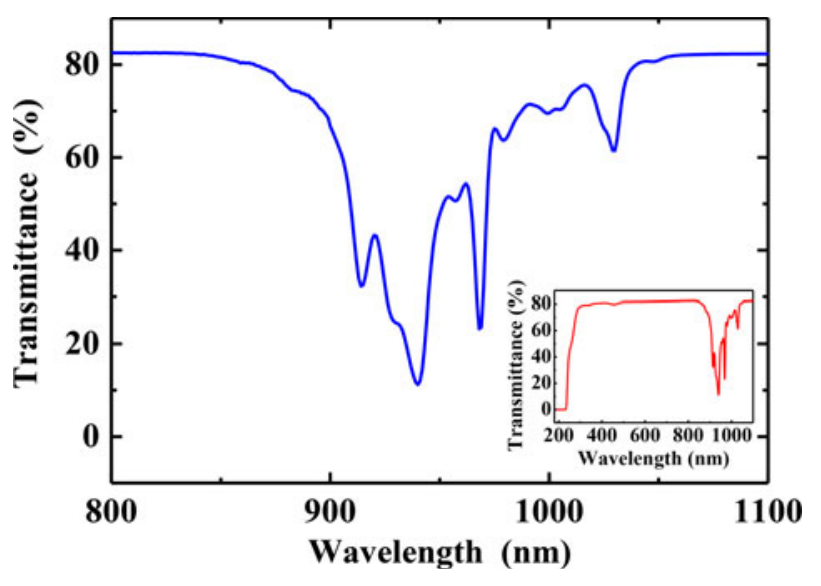

Figure 2. The transmittance curve of the YAG/Yb:YAG/YAG ceramic from 800 to $1100 \mathrm{~nm}$. The inset is the transmittance curve from 200 to $1100 \mathrm{~nm}$.

in the visible range; both of the two undoped layers and the doped interlayer in the unannealed ceramic are affected by lattice defects caused by oxygen vacancies. The doped interlayer exhibits a darker shade of green due to the effects of lattice defects as well as ' $\mathrm{F}$ ' color centers caused by ion transformations from $\mathrm{Yb}^{3+}$ to $\mathrm{Yb}^{2+[29,30]}$. The annealed ceramic was completely transparent, which can be seen from the image. The optical transmittance of the ceramic from 200 to $1100 \mathrm{~nm}$ is shown in Figure 2. It can be seen that the transmittance is as high as $83 \%$ from 400 to $800 \mathrm{~nm}$ and attains a higher value around $1100 \mathrm{~nm}$. From 800 to $1100 \mathrm{~nm}$, there are three strong absorption peaks around 915,940 and $970 \mathrm{~nm}$, which correspond to the conventional broadband absorption spectrum of $\mathrm{Yb}^{3+}$ ions. The strongest absorption rate reaches $90 \%$ at $940 \mathrm{~nm}$, which corresponds to the energy transition between two manifolds of $\mathrm{Yb}^{3+}$ ions.

In order to test the performance of the ceramic laser, a folded laser cavity was built, as schematically shown in Figure 3. The total cavity length was $450 \mathrm{~mm}$, which was pumped by a high-brightness fiber-pigtailed diode laser with a maximum output power of $30 \mathrm{~W}$ at $974 \mathrm{~nm}$. The core diameter of the fiber was $100 \mu \mathrm{m}$, and the numerical 


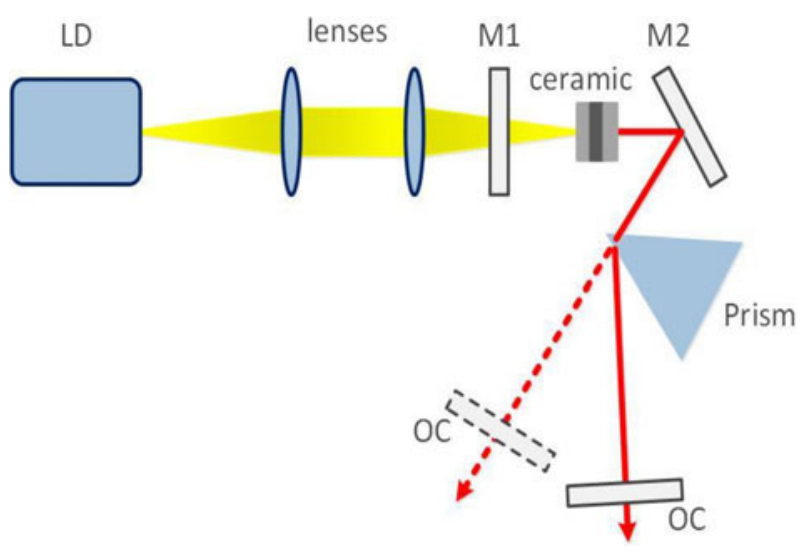

Figure 3. The experimental setup of the laser cavity.

aperture was 0.22. Two lenses were employed to build a 1:1 imaging system for efficient focusing of the pump beam into the surface of the ceramic sample with a spot size of about $100 \mu \mathrm{m}$. The size of the ceramic used in our experiment was $5 \mathrm{~mm} \times 5 \mathrm{~mm} \times 4 \mathrm{~mm}(X \times Y \times Z)$ and the doping concentration of $\mathrm{Yb}^{3+}$ ions was 5 at.\%. The ceramic was wrapped with indium foil and fixed tightly into a three-dimensional adjustment stage. The temperature of the gain medium was controlled at $14{ }^{\circ} \mathrm{C}$ by a watercooled copper heat sink. We adopted a stable three-mirror folded resonator. The resonator consisted of one dichroic input coupler (M1), one folding mirror (M2) with a radius of curvature of $300 \mathrm{~mm}$ and one output coupler (OC). The dichroic mirror M1 and folding mirror M2 were antireflection coated at $976 \mathrm{~nm}$ and high-reflection coated in a wavelength range from 1020 to $1120 \mathrm{~nm}$. Three OCs with different transmissions of $T=2 \%, T=5 \%$, and $T=10 \%$ from 1020 to $1120 \mathrm{~nm}$ were used to measure the laser performance. The output $\mathrm{CW}$ laser was monitored by a fiber spectrometer and a power meter.

In the process of adjusting the laser, we found that the output power hardly changed when we moved the focus spot of the pumping laser diode on the ceramic by using the three-dimensional adjustment frame $(X \times Y)$, which implied uniform distribution of the ceramic. Figure 4(a) shows the $\mathrm{CW}$ laser spectrum of the YAG/5 at.\% Yb:YAG/YAG ceramic. The output wavelength was about $1031 \mathrm{~nm}$ with a full width at half maximum (FWHM) of over $3 \mathrm{~nm}$. The CW laser oscillation could be observed by the fiber spectrometer with a threshold incident pump power of $0.45 \mathrm{~W}$ when using the $T=2 \%$ OC. In order to optimize the laser performance of the ceramic laser, we monitored the output power obtained using OCs with $T=2 \%, T=5 \%$, and $T=10 \%$, as shown in Figure 4(b). The maximum output power of $3.2 \mathrm{~W}$ was obtained when using an OC with $T=10 \%$ with an incident pump power of $26.6 \mathrm{~W}$, corresponding to a slope efficiency and optical conversion efficiency of $20.4 \%$ and $12.0 \%$, respectively. Once the incident pump power exceeded 20 $\mathrm{W}$, the output power tended to be unchanged with increase of pump power. This was mainly caused by the wavelength drift of the incident pump laser away from the narrow absorption bandwidth of $976 \mathrm{~nm}$ for the gain media due to the temperature change of the diode laser. By employing a pump laser with a stable wavelength and improving the cavity structure, a higher output power could be expected.

To further characterize the wavelength tunability of this YAG/Yb:YAG/YAG ceramic laser, we tuned the laser wavelength by inserting an SF57 Brewster prism into the resonator. It was located between the folded mirror and the $\mathrm{OC}$ at the Brewster angle. Under $5 \mathrm{~W}$ incident pump power, the Yb:YAG laser could be tuned from 1021 to $1058 \mathrm{~nm}$, with a maximum output power of $178 \mathrm{~mW}$ at $1031 \mathrm{~nm}$, as shown in Figure 5. The tuning curve was continuous and smooth, and the broadband tunability indicated the possibility of ultrashort femtosecond pulse generation by such a composite ceramic. The coating of all cavity mirrors had a reflection range starting from $1020 \mathrm{~nm}$; by employing a reflection coating with a lower cut-off wavelength, laser oscillation at wavelengths shorter than $1020 \mathrm{~nm}$ would be expected.

\section{Conclusion}

In summary, a continuously tunable YAG/Yb:YAG/YAG composite ceramic laser was demonstrated in a three-mirror

(b)

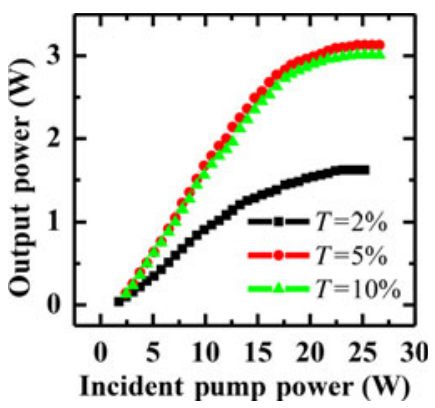

Figure 4. (a) The typical output laser spectrum and (b) the output power of the YAG/Yb:YAG/YAG multilayered ceramic laser using OCs with $T=2 \%$, $T=5 \%$, and $T=10 \%$. 


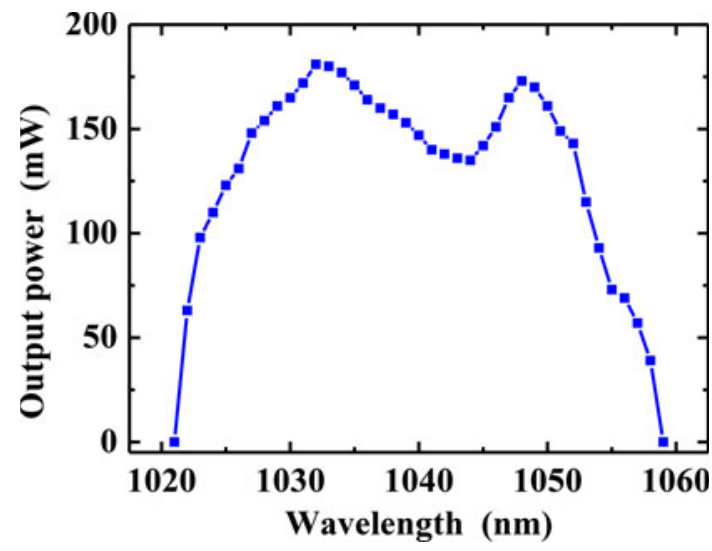

Figure 5. The tuning curve obtained with an intracavity dispersive prism for the $\mathrm{YAG} / 5$ at.\% $\mathrm{Yb}$ :YAG/YAG ceramic laser.

folded cavity. The multilayer composite ceramic with a sandwich structure was fabricated by the non-aqueous tape-casting method. At $26.6 \mathrm{~W}$ incident pump power, a $3.2 \mathrm{~W}$ maximum average output power of the $\mathrm{CW}$ laser was achieved at $1031 \mathrm{~nm}$ with a slope efficiency of $20.4 \%$. The minimum threshold incident pump power for laser oscillation was $0.45 \mathrm{~W}$. By inserting an SF57 prism in the laser cavity, a smooth tunable curve from 1021 to $1058 \mathrm{~nm}$ was obtained. Such multilayer composite ceramics support different doping concentrations, with high transmission efficiency from visible to infrared and continuous broadband wavelength tunability. Our results demonstrated that composite ceramics fabricated by the tape-casting technique could be tailored with flexible structures and improved heat dissipation, which are desirable qualities for the next generation of high-power ultrashort solid-state lasers.

\section{Acknowledgements}

This work was supported by the Major Program of the National Natural Science Foundation of China (Grant No. 50990301) and the Project for Young Scientists Fund of the National Natural Science Foundation of China (Grant Nos. 51002172 and 51302298).

\section{References}

1. J. Sanghera, W. Kim, G. Villalobos, B. Shaw, C. Baker, J. Frantz, B. Sadowski, and I. Aggarwal, Opt. Mater. 35, 693 (2013).

2. W. B. Liu, Y. P. Zeng, L. Wang, Y. Q. Shen, B. X. Jiang, J. Li, D. Zhang, and Y. B. Pan, Laser Phys. 22, 1622 (2012).
3. J. Akiyama, Y. Sato, and T. Taira, Opt. Lett. 35, 3598 (2010).

4. W. Liu, Y. Zeng, J. Li, Y. Shen, Y. Bo, N. Zong, P. Wang, Y. Xu, J. Xu, D. Cui, Q. Peng, Z. Xu, D. Zhang, and Y. Pan, J. Alloys Compd. 527, 66 (2012).

5. A. Ikesue, T. Kinoshita, K. Kamata, and K. Yoshida, J. Am. Ceram. Soc. 78, 1033 (1995).

6. J. Lu, J. Lu, T. Murai, K. Takaichi, T. Uematsu, K. Ueda, H. Yagi, T. Yanagitani, and A. A. Kaminskii, Jpn. J. Appl. Phys. 40, L1277 (2001).

7. J. Lu, K. Takaichi, T. Uematsu, A. Shirakawa, M. Musha, K. Ueda, H. Yagi, T. Yanagitani, and A. A. Kaminskii, Appl. Phys. Lett. 81, 4324 (2002).

8. T. Taira, A. Mukai, Y. Nozawa, and T. Kobayashi, Opt. Lett. 16, 1955 (1991).

9. W. Li, Q. Hao, H. Zhai, H. Zeng, W. Lu, G. Zhao, L. Zheng, L. Su, and J. Xu, Opt. Express 15, 2354 (2007).

10. H. Yoshioka, S. Nakamura, T. Ogawa, and S. Wada, Opt. Express 17, 8919 (2009).

11. A. Shirakawa, K. Takaichi, H. Yagi, J. Bisson, J. Lu, M. Musha, K. Ueda, T. Yanaqitani, T. Petrov, and A. Kaminskii, Opt. Express 11, 2911 (2003).

12. A. Shirakawa, K. Takaichi, H. Yagi, M. Tanisho, J. F. Bisson, J. Lu, K. Ueda, T. Yanagitani, and A. A. Kaminskii, Laser Phys. 14, 1375 (2004).

13. X. J. Cheng, B. X. Jiang, L. Li, J. L. Wang, Z. G. Yang, W. B. Cheng, X. C. Shi, and Y. B. Pan, Laser Phys. 22, 652 (2012).

14. H. Y. Zhu, C. W. Xu, J. Zhang, D. Y. Tang, D. W. Luo, and Y. M. Duan, Laser Phys. Lett. 10, 75802 (2013).

15. C. W. Xu, D. Y. Tang, H. Y. Zhu, and J. Zhang, Laser Phys. Lett. 10, 95702 (2013).

16. M. Tsunekane and T. Taira, Appl. Phys. Lett. 90, 121101 (2007).

17. H. Zhou, W. Li, K. Yang, N. Lin, B. Jiang, Y. Pan, and H. Zeng, Opt. Express 20, A489 (2012).

18. A. A. Kaminskii, S. N. Bagayev, K. Ueda, K. Takaichi, H. Yagi, and T. Yanagitani, Laser Phys. Lett. 3, 124 (2006).

19. Q. Hao, W. Li, H. Zeng, Q. Yang, C. Dou, H. Zhou, and W. Lu, Appl. Phys. Lett. 92, 211106 (2008).

20. Q. Hao, W. Li, H. Pan, X. Zhang, B. Jiang, Y. Pan, and H. Zeng, Opt. Express 17, 17734 (2009).

21. N. Lin, W. Li, Y. Zhou, Y. Shi, M. Yan, K. Yang, J. Zhao, X. Yang, and H. Zeng, Laser Phys. Lett. 10, 15103 (2013).

22. A. Pirri, M. Vannini, V. Babin, M. Niki, and G. Toci, Laser Phys. 23, 95002 (2013).

23. A. Ikesue, Y. L. Aung, T. Taira, T. Kamimura, K. Yoshida, and G. L. Messing, Ann. Rev. Mater. Res. 36, 397 (2006).

24. A. Ikesue and Y. L. Aung, Nat. Photon. 2, 721 (2008).

25. J. Li, Y. Pan, Y. Zeng, W. Liu, B. Jiang, and J. Guo, Int. J. Refract. Met. Hard Mater. 39, 44 (2013).

26. F. Tang, Y. Cao, J. Huang, H. Liu, W. Guo, and W. Wang, J. Am. Ceram. Soc. 95, 56 (2012).

27. X. Ba, J. Li, Y. Pan, L. Jing, B. Jiang, W. Liu, H. Kou, and J. Guo, J. Rare Earths 31, 507 (2013).

28. Q. Liu, M. Gong, F. Lu, W. Gong, and C. Li, Opt. Lett. 30, 726 (2005).

29. P. Yang, P. Deng, and Z. Yin, J. Lumin. 97, 51 (2002).

30. X. Xu, Z. Zhao, P. Song, G. Zhou, J. Xu, and P. Deng, J. Opt. Soc. Am. B 21, 543 (2004). 